\title{
Physical conditions and chemistry of molecular gas in galactic centers
}

\author{
Susanne Aalto \\ Department of Earth and Space Sciences, Chalmers University of Technology \\ Onsala Space Observatory, SE-439 92 Onsala, Sweden \\ email: saalto@chalmers.se
}

\begin{abstract}
Studying the molecular phase of the interstellar medium in galaxy nuclei is fundamental for the understanding of the onset and evolution of star formation and the growth of supermassive black holes. We can use molecules as observational tools exploiting them as tracers of chemical, physical and dynamical conditions. The molecular physical conditions in galaxy centers show large variety among galaxies, but in general the average gas densities (traced by e.g. $\mathrm{HCN}$ ) and temperatures (probed by e.g. $\mathrm{H}_{2} \mathrm{CO}, \mathrm{NH}_{3}$ ) are greater than in their disks. Molecular gas and dust is being funneled to the centers of galaxies by spiral arms, bars, and interactions - and one example of this is the minor merger NGC1614. Gas surface densities are also greater in galaxy nuclei and in extreme cases they become orders of magnitudes larger than what we find in the center of our own Milky Way. We can use IR excited molecular emission to probe the very inner regions of galaxies with deeply obscured nuclei where $N\left(\mathrm{H}_{2}\right)>10^{24} \mathrm{~cm}^{-2}$ - for example the luminous infrared galaxy (LIRG) NGC4418. Abundances of key molecules such as $\mathrm{HCN}, \mathrm{HCO}^{+}, \mathrm{HNC}, \mathrm{HC}_{3} \mathrm{~N}, \mathrm{CN}, \mathrm{H}_{3} \mathrm{O}^{+}$are important tools in identifying the nature of buried activity and its evolution. Standard astrochemical scenarios (including X-ray Dominated regions (XDRs) and Photon Dominated Regions (PDRs)) are briefly discussed in this review and how we can use molecules to distinguish between them. High resolution studies are often necessary to separate effects of excitation and radiative transfer from those of chemistry - one example is absorption and effects of stimulated emission in the ULIRG Arp220. The nuclear activity in luminous galaxies often drives outflows and winds and in some cases molecular gas is being entrained in the outflows. Sometimes the molecular gas is carrying the bulk of the momentum. We can study the structure and physical conditions of the molecular gas to constrain the mass outflow rates and the evolution and nature of the driving source and two examples are discussed here: NGC1377 and Mrk231.
\end{abstract}

Keywords. galaxies: evolution — galaxies: ISM — galaxies:active — radio lines: ISM — ISM: molecules — ISM:abundances — astrochemistry

\section{Introduction}

Molecular emission and absorption are very useful tools to study the nature and evolution of nuclear activity - in particular of luminous and ultraluminous infrared galaxies (LIRGs and ULIRGs). Molecules are fundamentally important since they serve as fuel for the evolution of galaxies through star formation and the growth of supermassive black holes (SMBHs). The discovery of molecular gas in large scale galactic outflows also suggests that molecules play a part in the turning-off and regulation of starbursts and AGNs. Furthermore, mm and submm emission can penetrate highly obscured regions allowing us to probe the dusty nuclei of LIRGs and ULIRGs revealing the nature of the buried activity.

In this short review, the driving of molecular gas towards galaxy centers is briefly discussed in Section 2. In Section 3 some very general results on physical conditions and ISM structure in galaxy nuclei are discussed - together with a look at Compact 


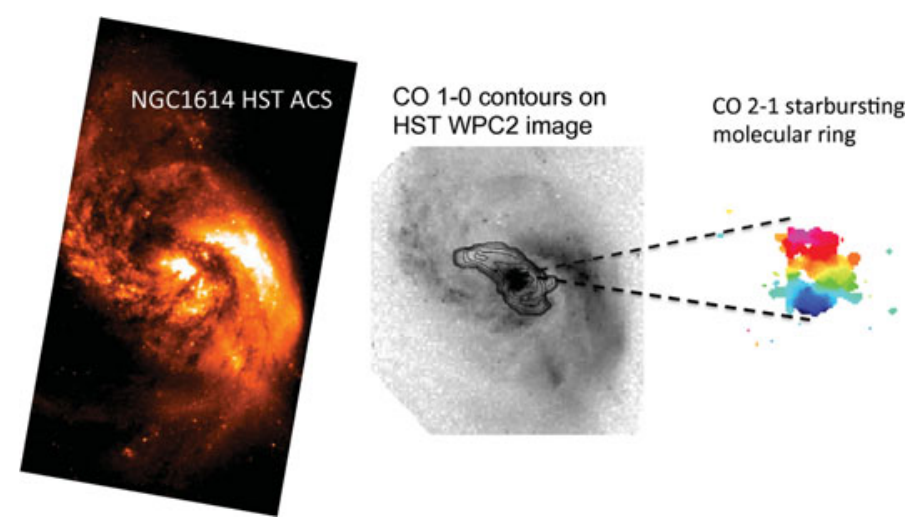

Figure 1. Molecular gas in the starburst minor merger NGC1614: Left: Hubble ACS image of NGC1614. Center: OVRO CO $J=(1-0)$ contours overlayed on a Hubble WPC2 image of NGC1614 (Olsson et al. 2010). Right: SMA CO $J=(2-1)$ velocity field of the starbursting molecular ring (König et al. 2013). [A COLOR Version is AVAILABle onLine.]

Obscured Nuclei (CONs) and vibrationally excited molecules. The use of molecules as tracers of extragalactic astrochemistry is introduced in Section 4 together with a selection of useful molecular lines. In Section 5, global molecular line ratios and examples of spectral scans are discussed. In Section 6, the importance of studying molecular chemistry and excitation at high resolution is discussed and in Section 7 new interferometric results on molecular gas in large scale outflows and winds are presented.

\section{Driving the gas to the center}

How does the gas get to the centers of galaxies? In general, processes such as spiral arms, bars and galaxy interactions/mergers cause gas inflow toward galaxy nuclei. The gas collects in disks (sometimes counter-rotating), rings, mini-bars, Compact Obscured Nuclei (CONs) and feeds AGN and/or starburst activity - which in turn drives outflows and winds. A discussion of bar-driven inflow can be found in the chapter by K. Sakamoto in this book. Below I describe an example of interaction driven inflow in the luminous minor merger NGC1614. Here, tidal gas gas is returning along a minor axis dust lane (which likely is a polar ring) and collecting in a molecular starburst ring in the inner region of the merger.

\subsection{The "ring of fire" in NGC1614}

The most intense CO $J=(2-1)$ emission of the starburst minor merger NGC1614, occurs in a partial ring with $\approx 230$ pc radius around the center with an extension to the northwest into the dust lane that contains diffuse molecular gas. Ten giant molecular associations (GMAs) are found in the ring, which have an integrated molecular mass of $8 \times 10^{8} \mathrm{M}_{\odot}$. The interferometric observations filter out a large part of the CO $J=(2-1)$ emission which would be recovered with shorter spacings. This suggests that the bulk of the molecular gas in NGC1614 is diffuse and that GMAs only exist near and within the circumnuclear ring. The molecular ring is uneven with most of the mass on the western side, which also contains GMAs extending into a pronounced tidal, minor axis dust lane (Figure 1).

\subsubsection{Feeding the center: minor axis dust lane - a polar ring?}

The northwest extension of the ring seems to be a cosmic "umbilical cord" that is feeding molecular gas associated with the dust lane and tidal debris into the nuclear ring, which contains the bulk of the starburst activity. The ring structure in the center 


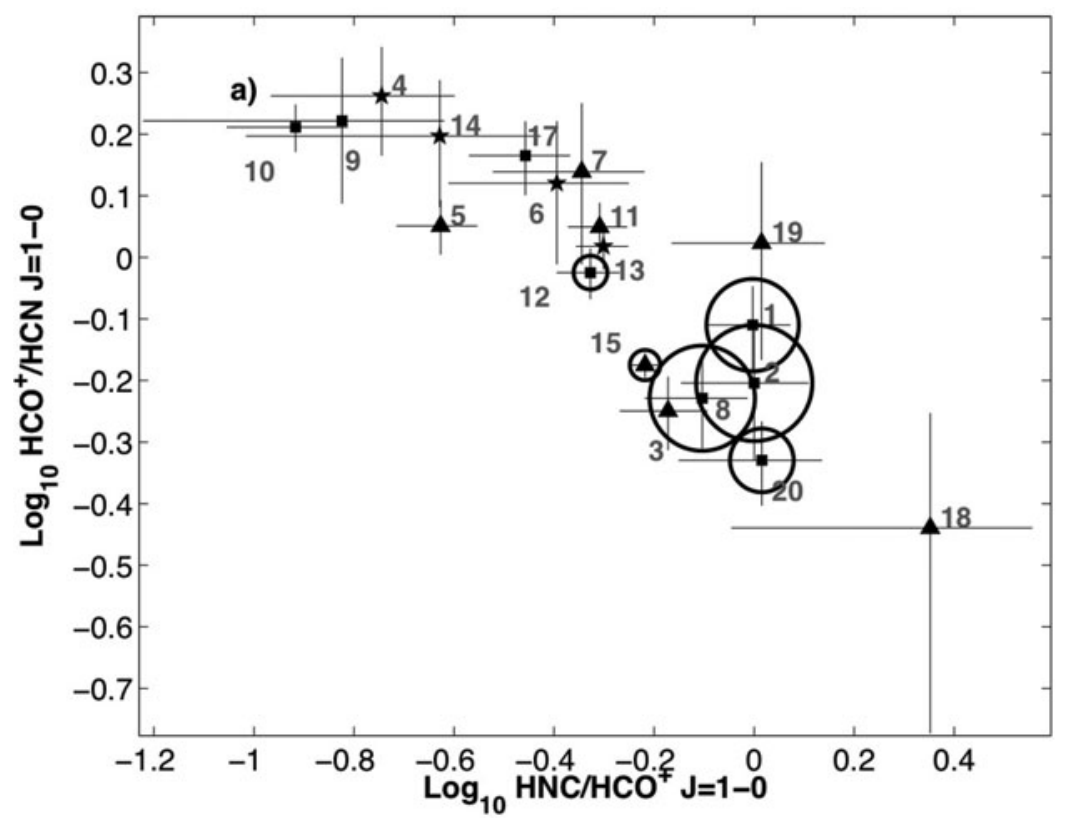

Figure 2. Plot of the $\mathrm{HCO}^{+} / \mathrm{HCN}$ vs. the $\mathrm{HNC} / \mathrm{HCO}^{+} J=(1-0)$ line ratio for a sample of luminous LIRGs, ULIRGs and AGNs (Costagliola et al. 2011). The circles indicate galaxies with $\mathrm{HC}_{3} \mathrm{~N} J=(10-9)$ detections where the diameters of the circles are proportional to the $\mathrm{HC}_{3} \mathrm{~N} J=(10-9) / \mathrm{HCN} J=(1-0)$ line ratio. Error bars show $1 \sigma$ uncertainties. The $\mathrm{HC}_{3} \mathrm{~N}$ luminous galaxies are also $\mathrm{HNC}$-bright with respect to $\mathrm{HCO}^{+}$and $\mathrm{HCN}$. The lines were observed simultaneously with the IRAM 30m EMIR receiver.

may be the final resting place of tidal gas that is being transported from large $r$ s along the dust lane/polar ring into the center of NGC1614. The presence of numerous GMAs suggests that an orbit-crowding or resonance phenomenon occurs near the ring - helping to turn diffuse gas into self-gravitating GMAs. The net result of this merger therefore very likely increases the central concentration of stellar mass in the NGC1614 remnant giant system (König et al. 2013).

\section{Physical conditions and ISM structure}

The CO $J=(1-0)$ line is often used to trace $\mathrm{H}_{2}$ mass (e.g. Paglione et al. 2001; Wada \& Tomisaka 2005; Narayanan et al. 2012) and gas dynamics. The line intensity ratio between $\mathrm{CO}$ and the polar molecule $\mathrm{HCN}$ is a popular measure of the mass fraction of dense $\left(n>10^{4} \mathrm{~cm}^{-3}\right)$ molecular gas (e.g. Gao \& Solomon 2004). In general, gas densities (by mass) in galaxy nuclei are greater than those in the disks of the galaxies which is reflected in an elevated $\mathrm{HCN} / \mathrm{CO} J=(1-0)$ line ratio (e.g. Helfer \& Blitz 1993; Gao \& Solomon 2004; Curran et al. 2001). Also the CO $/{ }^{13} \mathrm{CO} J=(1-0)$ line ratios are elevated in galaxy nuclei (e.g. Aalto et al. 1995, 1997). Isotopic variants (isotopomers) e.g. ${ }^{13} \mathrm{C},{ }^{18} \mathrm{O},{ }^{15} \mathrm{~N}$ of species can help measure optical depth variations and thus map out the structure of the interstellar medium and find changes in the physical conditions. For example, variations in the $\mathrm{CO} /{ }^{13} \mathrm{CO} J=(1-0)$ intensity ratio that map the dynamical effects on clouds in spiral arms and bars and/or effects of temperature gradients (e.g. Meier et al. 2000; Tosaki et al. 2002; Aalto et al. 1997, 2010; Hirota et al. 2010). Globally the $\mathrm{CO} /{ }^{13} \mathrm{CO} J=(1-0)$ intensity ratio increases rather strongly with dust temperature, an effect that can largely be explained as a gas temperature effect (e.g. Aalto et al. 1995; Costagliola et al. 2011). Studying isotopic variants will also give important information on 
stellar nucleosynthesis and the number of stellar generations the ISM has gone through. In general for galaxies, $\mathrm{CO} /{ }^{13} \mathrm{CO} J=(1-0)$ line ratios increase from GMC-like ratios of $5-7$ in the disks to typical values of 10-15 in galaxy centers and even more extreme ratios $>30$ in the inner regions of hot luminous mergers (e.g. Aalto et al. 1995).

The bright $\mathrm{HCN}$ and faint (low- $J){ }^{13} \mathrm{CO}$ emission can be explained by a warm, high pressure molecular interstellar medium where most of the mass resides in dense $\left(n>10^{4}\right.$ $\left.\mathrm{cm}^{-3}\right)$ clouds/clumps while most of the volume is at low density $\left(n=10^{2}-10^{3} \mathrm{~cm}^{-3}\right)$, where ${ }^{13} \mathrm{CO}$ emission will be faint (e.g. Aalto et al. 1995). The reduced ${ }^{13} \mathrm{CO}$ emission can either be a result of a low-to-moderate optical depth in the low-density clouds and/or an effect of the high $(>50 \mathrm{~K})$ temperature, which will depopulate the lower levels and reduce the optical depths of the $J=(1-0)$ lines. This will also make the ${ }^{13} \mathrm{CO}$ more vulnerable to photo dissociation - further reducing the ${ }^{13} \mathrm{CO}$ luminosity. In addition, stellar nucleosynthesis will impact the $\mathrm{C} /{ }^{13} \mathrm{C}$ ratio - and other isotopic ratios.

Molecules that more directly can be used to measure gas kinetic temperature are $\mathrm{NH}_{3}$ and $\mathrm{H}_{2} \mathrm{CO}$. The relative populations of the $K_{a}$ ladders of the (slightly) asymmetric top molecule $\mathrm{H}_{2} \mathrm{CO}$ are generally governed by collisions and inter-ladder line ratios are thus generally good tracers of the gas kinetic temperature. Mühle et al. (2007) observed para- $\mathrm{H}_{2} \mathrm{CO}$ in the starburst M82 to deduce the presence of $200 \mathrm{~K}$ molecular gas. The inversion-rotation transitions of $\mathrm{NH}_{3}$ may also be used to accurately determine gas kinetic temperature (e.g. Martin \& Ho 1986). More recently, Mangum et al. (2013) used $\mathrm{NH}_{3}$ as a temperature probe of the nearby starburst galaxy NGC253 and found several temperature components in the molecular gas ranging from $\approx 40 \mathrm{~K}$ to $>150 \mathrm{~K}$ with a spatial northeast to southwest gradient.

There is a large variety in molecular physical conditions, structure and extent in galaxy centres on scales similar to those of the central molecular zone (400 - 500 pc) of the Milky Way. NGC253 is often suggested to have similar physical and chemical conditions as the Milky Way central molecular zone (CMZ). $\mathrm{H}_{2} \mathrm{CO}$ studies of the inner 100 by $20 \mathrm{pc}$ (Ao et al. 2013) of the Galactic center find warm temperatures with $T_{\mathrm{k}}$ for individual molecular clouds ranging from $50 \mathrm{~K}$ to $>100 \mathrm{~K}$ and the clouds with highest temperature are found near the nucleus. For the molecular gas outside the dense clouds, the average $T_{\mathrm{k}}$ is $65 \pm 10 \mathrm{~K}$. The high temperatures of molecular clouds on large scales in the GC region may be driven by turbulent energy dissipation and/or cosmic-rays - a similar scenario to the one suggested for NGC253.

\subsection{Compact obscured nuclei}

In some cases gas funneling processes lead to extreme molecular gas and dust concentrations rendering the central regions opaque. The resulting Compact Obscured Nuclei (CONs) may have $L_{\mathrm{bol}}>10^{9} \mathrm{~L}_{\odot}$ emerging from inside $d<100 \mathrm{pc}$, dusty $\left(A_{\mathrm{v}} 100-\right.$ $1000 \mathrm{mag})$ cores. Thus, the nature of the source powering the nuclear activity cannot be determined with conventional methods such as optical and IR lines - even X-rays may fail because of the nuclear Compton thick shroud. It is important to determine if the powering source is an accreting black hole or a compact starburst (or a combination) since it has profound impact on the understanding of galaxy evolution - both near and far. The fraction of AGN luminosity, for example, obscured by dust and re-emitted in the mid-IR is critical for understanding AGN evolution, unification, and parsec-scale AGN physics. Highly obscured nuclei are also in a transition phase towards unobscured starburst or AGN activity where the feedback mechanisms (outflows and radiation) are key processes in galaxy evolution.

Some galaxies with CONs, such as NGC4418 (Figure 3), have extreme gas surface densities compared to that of the CMZ. In NGC4418, $10^{8} \mathrm{M}_{\odot}$ is crammed into a structure of less than 20 pc in diameter (Sakamoto et al. 2013) while a similar mass (or less) is 

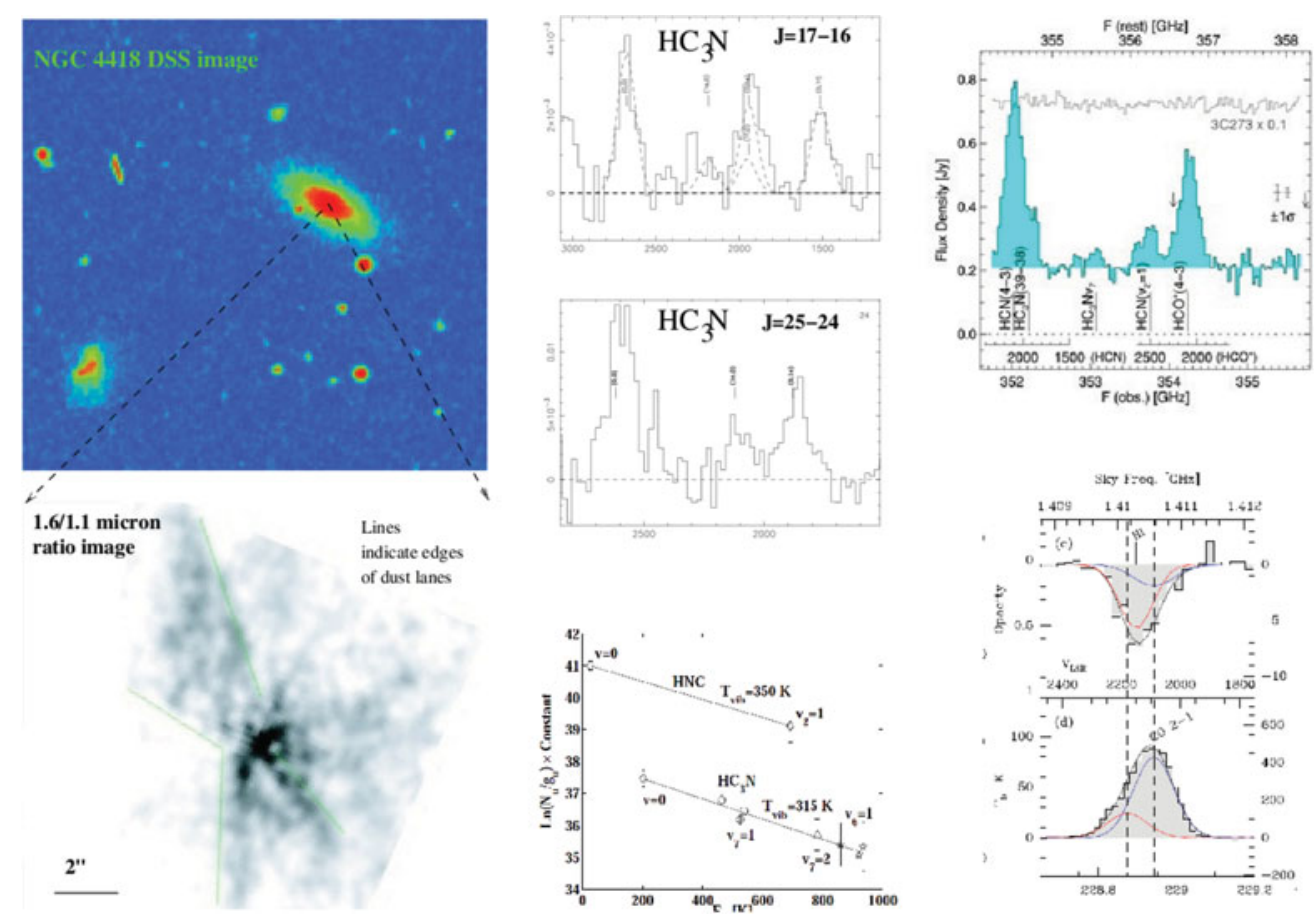

Figure 3. Upper left: DSS image of the LIRG NGC4418. Lower left: NICMOS NIR image of the ratio of the 1.6/1.1 $\mu \mathrm{m}$ continuum revealing the dusty interior of NGC4418 (Evans et al. 2003). Upper center: Two spectra of intense $\mathrm{HC}_{3} \mathrm{~N}$ emission - rotational and vibrational - from NGC4418 and below the spectra is a rotational diagram of $\mathrm{HC}_{3} \mathrm{~N}$ and $\mathrm{HNC}$. Upper right: SMA spectrum with $\mathrm{HCN} J=(4-3)$ and $\mathrm{v}_{2}=1, \mathrm{HCO}^{+} J=(4-3), \mathrm{HC}_{3} \mathrm{~N}(39-38)$ and $\mathrm{v}_{7}=1$ (Sakamoto et al. 2010). Lower right: Merlin Hi absorption (top) and SMA CO $J=(2-1)$ emission showing evidence for infalling gas towards the nucleus of NGC4418 (Costagliola et al. 2013). [A COLOR VERSION IS AVAILABLE ONLINE.]

distributed on a scale more than an order of magnitude larger in the Milky Way CMZ (Martin et al. 2004). The bulk temperature of this gas in NGC4418 exceeds $80 \mathrm{~K}$ and the excitation of molecules are heavily affected by the intense IR radiation field.

\subsection{Molecular excitation - IR pumping of molecules}

When we interpret $\mathrm{mm}$ and submm molecular spectra from external galaxies we often assume that the excitation is dominated by collisions with $\mathrm{H}_{2}$. However, there are also other possible mechanisms including IR radiative excitation where molecules absorb IR continuum corresponding to (e.g.) their bending modes. It is possible that this IR-pumping may affect the excitation of the rotational levels in the vibrational ground state. Thus, to correctly interpret the molecular emission, we must examine its excitation. For example, both HCN and HNC can absorb IR-photons to the bending mode (its first vibrational state) and then it decays back to the ground state via its P-branch $(\nu=1-0, \Delta J=+1)$ or R-branch $(\nu=1-0, \Delta J=-1)$. In this way, a vibrational excitation may produce a change in the rotational state in the ground level and can be treated (effectively) as a collisional excitation in the statistical equations. Thus, IR pumping excites the molecule to the higher rotational level by a selection rule $\Delta J=2$. For $\mathrm{HNC}$, the bending mode occurs at $\lambda=21.5 \mu \mathrm{m}\left(464.2 \mathrm{~cm}^{-1}\right)$ with an energy level $h \nu / k=669 \mathrm{~K}$ and an $A$ coefficient of $A_{\mathrm{IR}}=5.2 \mathrm{~s}^{-1}$. For $\mathrm{HCN}$ the mode occurs at $\lambda=14 \mu \mathrm{m}\left(713.5 \mathrm{~cm}^{-1}\right)$, energy level $h \nu / k=1027 \mathrm{~K}$ and $A_{\mathrm{IR}}=1.7 \mathrm{~s}^{-1}$. The pumping of HNC and HCN may 
start to become effective when the IR background reaches an optically thick brightness temperature of $T_{\mathrm{B}} \approx 50 \mathrm{~K}$ and $85 \mathrm{~K}$ respectively - and for gas densities below critical.

Furthermore, emission from rotational transitions within the vibrationally excited bands can be used to probe inside the optically thick dust cocoons in the nuclei of deeply obscured galaxies. The HCN molecule may reach large abundances in warm $(100$ - $500 \mathrm{~K})$ environments near embedded AGNs (e.g. Harada et al. 2010) and HCN vibrational transitions are ideal tracers of hot nuclear molecular gas. The first extragalactic detection of HCN $J=(3-2) \mathrm{v}_{2}=1 \mathrm{f}$ was in the LIRG NGC4418 (Figure 3) (Sakamoto et al. 2010) and recently, the line has been imaged in the ULIRG QSO Mrk 231 where it is tracing a 75 pc misaligned disk/torus around the AGN. The collisionally excited $v=0 \mathrm{HCN} J=(3-2)$ line is instead tracing the main stellar disk - and the powerful molecular outflow (Aalto et al. in preparation). The HCN $J=(4-3) v_{2}=1 \mathrm{f}$ line has also been detected in the LIRG AGN-candidate IRAS 20551 (Imanishi \& Nakanishi 2013). Rotational-vibrational lines of $\mathrm{HC}_{3} \mathrm{~N}$ and $\mathrm{HNC}$ have been detected in $\mathrm{NGC4418}$ where the vibrational temperatures $T_{\text {vib }}$ of $\mathrm{HCN}, \mathrm{HNC}$ and $\mathrm{HC}_{3} \mathrm{~N}$ are $200-400 \mathrm{~K}$, suggesting the presence of a "hot core" - heated either by an extremely compact young starburst or an embedded AGN. The size of this core has been determined to $r<5$ pc (Costagliola \& Aalto 2010; Sakamoto et al. 2010; Costagliola et al. 2013; Sakamoto et al. 2013). Mills et al. (2013) detect $\mathrm{HCN} J=(4-3), v_{2}=1$ emission in the circumnuclear disk (CND) of the Milky Way. This appears to be from only one clump in the CND while it seems like in galaxies like NGC4418 a larger fraction of the circumnuclear gas is affected by IR excitation.

\section{Extragalactic astrochemistry}

Astrochemistry offers an additional new tool to study galaxy evolution, in particular in deeply dust-obscured objects. The radiative and dynamical environment will impact both gas physical conditions and its chemistry. We may therefore develop astrochemical models that we can use to understand the evolution of molecular gas in galaxies. There are a number of standard scenarios often referred to when we discuss extragalactic astrochemistry:

(a) Photon (or Photo) dominated region (PDR) Regions are affected by farultraviolet (UV) photons (energy $\mathrm{h} \nu=6-13.6 \mathrm{eV}$ ). Bulk temperatures are expected to be moderate $(20-50 \mathrm{~K})$ while surface temperatures may be large $(300-1000 \mathrm{~K})$ due to the layered structure of the PDR. Photo-chemical processes dominate (e.g. Hollenbach \& Tielens 1997) but the penetration of the UV photons is limited by dust and, as $A_{\mathrm{V}}$ increases, cosmic rays take over as the dominant source of ionization reactions. The heating occurs through the photoelectric effect on grains and PAHs and collisional deexcitation of $\mathrm{H}_{2}$.

(b) X-ray dominated region (XDR) These regions are signified by large bulk temperatures $>100 \mathrm{~K}$ and a chemical structure typical of the special ion-neutral chemistry triggered by the irradiation of X-rays (with $\mathrm{h} \nu=1-100 \mathrm{keV}$; e.g. Maloney et al. 1996; Lepp \& Dalgarno 1996; Meijerink \& Spaans 2005). The X-rays provide a larger penetration depth than in PDRs and a more efficient heating mechanism. The ionization occurs through primary $\mathrm{X}$-rays and secondary photo electrons. The heating occurs through Coulomb heating, $\mathrm{H}_{2}$ ionization, $\mathrm{H}_{2}$ vibrational excitation, and dissociative excitation. The ionization rate is high (from the secondary photo electron).

(c) Cosmic ray dominated region (CDR or CRDR) Regions of elevated ( $>10^{3} \times$ Galactic value) cosmic ray energy density (e.g. Suchkov et al. 1993; Meijerink et al. 2011; Bayet et al. 2011) primarily originating from supernovae.

(d) Dense shielded regions In warm, dusty and dense regions, that are relatively shielded from harmful radiation, hot core-like chemistry may dominate with temperatures 
ranging from 50 to $500 \mathrm{~K}$ (e.g. Nomura \& Millar 2004; Viti 2005). Icy grain mantles are released affecting chemistry and the intense infrared (IR) radiation fields impact the molecular excitation (Costagliola \& Aalto 2010; Sakamoto et al. 2010). Formation and survival of complex species result in rich chemistry.

(e) Mechanically dominated region The chemistry reflects the speed of the shock and thus the level of grain processing (e.g. Usero et al. 2007; Viti et al. 2011; Kazandjian et al. 2012). Milder shocks result in the icy mantles coming off - and in more violent shocks the grain cores may be affected. Shock temperatures can be very high ranging from $100 \mathrm{~K}$ (C-shocks) up to a few thousand K (J-shocks). The dissipation of turbulence act on the bulk of the gas and may heat the gas to $\mathrm{C}$-shock temperatures.

It is likely that within one resolving beam we have multiple scenarios represented. However, we have a large variety of spectral tools at our disposal allowing us to probe a wide range of physical environments, temperatures, and densities of the interstellar medium. Combining molecular species and transitions with spatial resolution and sensitivity will enable us to disentangle chemical scenarios and to separate effects of excitation and radiative transfer from those of chemistry.

\subsection{Some useful molecular emission lines}

A summary of molecular species detected in extragalactic sources can be found in Martín et al. (2011). By 2011, a total of 46 species and 23 isotopic variants had been identified. With ALMA in full operation this list will grow significantly in the coming years. Intensity ratios of emission lines between species are often used to identify various astrochemical scenarios and/or physical conditions in the gas. Below is a list of a few popular molecular lines in astrochemistry.

- Molecular ions: $\mathbf{H C O}^{+}$and $\mathbf{H}_{3} \mathbf{O}^{+}$In the molecular cores around some AGNs elevated $\mathrm{HCN} / \mathrm{HCO}^{+} J=(1-0)$ intensity ratios have been found (e.g. Kohno 2003; Imanishi et al. 2009) and also in some ULIRGs (Graciá-Carpio et al. 2006). A suggested interpretation is that this is a chemical effect due to the presence of an XDR. However, theoretical models are not in agreement on whether the $\mathrm{HCO}^{+}$abundance is suppressed or enhanced relative to HCN in XDRs (e.g. Maloney et al. 1996; Meijerink \& Spaans $2005)$. Furthermore, $\mathrm{HCN} / \mathrm{HCO}^{+} J=(1-0)$ abundance ratios $>1$ are also expected in dense shielded gas (e.g. Aalto et al. 2007a) and in gas heated by shocks (e.g. Kazandjian et al. 2012). Thus, it is possible that $\mathrm{HCN} \mathrm{HCO}^{+} \mathrm{J}=(1-0)$ ratios are generally enhanced in compact molecular regions towards galaxy nuclei - regardless of the nature of the buried activity. For $\mathrm{H}_{3} \mathrm{O}^{+}$, models predict an order of magnitude greater abundances in XDRs than in PDRs. The first extragalactic $364 \mathrm{GHz}$ detections seem to support this notion (van der Tak et al. 2008; Aalto et al. 2011). It is however possible that elevated $\mathrm{H}_{3} \mathrm{O}^{+} / \mathrm{H}_{2} \mathrm{O}$ ratios are also consistent with CDRs - this requires further investigation. For example, Meijerink et al. (2011) suggest observing a combination of species, $\mathrm{OH}^{+}, \mathrm{OH}$, $\mathrm{H}_{2} \mathrm{O}^{+}, \mathrm{H}_{2} \mathrm{O}$ and $\mathrm{H}_{3} \mathrm{O}^{+}$, to distinguish between CDRs and XDRs. Several of these species have been studied in external galaxies by Herschel (e.g. van der Werf et al. 2010).

- Isomers: HNC In cold $(T<24 \mathrm{~K})$ gas $\mathrm{HNC} / \mathrm{HCN}$ abundance ratios are expected to be greater than unity while in dense, warmer gas and in shocked gas $X(\mathrm{HCN})>X(\mathrm{HNC})$ (Schilke et al. 1992). However, in XDRs and PDRs $X(\mathrm{HCN}) \gtrsim X(\mathrm{HNC})$ also in warm gas (Meijerink \& Spaans 2005) which complicates the use of the HCN/HNC abundance ratio as a tracer of gas temperature. Surveys reveal that global HCN/HNC $J=(1-0)$ intensity ratios in luminous galaxies often range between 1 and 6 (e.g. Aalto et al. 2002; Baan et al. 2010), but there are cases where the $\mathrm{HNC} / \mathrm{HCN} J=(3-2)$ intensity ratio exceeds unity (Aalto et al. 2007b, 2009). The cause for this "overluminosity" of HNC has been suggested to be either due to excitation and/or to effects of IR-pumping of HNC (see section on excitation above). In some XDR models the HNC abundances may also 
exceed those of $\mathrm{HCN}$ by a factor of two, but for this effect to lead to $I(\mathrm{HNC})>I(\mathrm{HCN})$ the optical depth of the lines has to be fairly low.

- Shielded gas: $\mathbf{H C}_{3} \mathbf{N}$ Surveys have revealed a subset of luminous galaxies with unusually bright $\mathrm{HC}_{3} \mathrm{~N} J=(10-9)$ emission compared to $\mathrm{HCN} J=(1-0)$ (Lindberg et al. 2011; Costagliola et al. 2011). $\mathrm{HC}_{3} \mathrm{~N}$ is destroyed by $\mathrm{UV}$ and particle radiation and in the Galaxy it can be found in high abundance in hot cores and in general in dense, warm and shielded gas. Interestingly, surveys reveal bright $\mathrm{HC}_{3} \mathrm{~N}$ line emission from LIRGs/ULIRGs with deep IR silicate absorption features and warm FIR colors suggesting a preference for dust obscured galaxies with high $A_{\mathrm{V}}$ and deeply buried (young?) activity.

- Radicals: CN In contrast to $\mathrm{HC}_{3} \mathrm{~N}$, enhancement of $\mathrm{CN}$ is expected in XDRs and in PDRs (e.g. Aalto et al. 2002; Baan et al. 2010; Meijerink \& Spaans 2005). The radical $\mathrm{CN}$ is also a tracer of dense gas, with a somewhat lower (by a factor of 5) critical density than HCN. Observations of the CN emission towards the Orion A molecular complex (Rodriguez-Franco et al. 1998) show that the morphology of the CN emission is dominated by the ionization fronts of the HiI regions. CN is also chemically linked to $\mathrm{HCN}$ via photodissociation. The abundance enhancement of $\mathrm{CN}$ over $\mathrm{HCN}$ is greater in an XDR (factors 40 - 1000) than in a PDR $(\mathrm{CN} / \mathrm{HCN}$ abundance ratio range from 0.5 to 2) (Lepp \& Dalgarno 1996; Meijerink \& Spaans 2005). Other radicals, such as CH, NO and $\mathrm{OH}$ are also more enhanced (relative to $\mathrm{HCN}$ ) in an XDR than in a PDR and the $\mathrm{CH} / \mathrm{HCN}$ column density, for example, may exceed $10^{3}$.

- Shock tracers: $\mathrm{SiO}, \mathrm{H}_{2} \mathrm{O}, \mathrm{HNCO}, \mathbf{C H}_{3} \mathbf{O H}$ Shocks can form $\mathrm{SiO}$ through the sputtering of $\mathrm{Si}$ from silicate grain cores, followed by reactions between the released $\mathrm{Si}$ and $\mathrm{O}_{2}$ or $\mathrm{OH}$ (Guillet et al. 2009). The shock must therefore be strong enough to get the $\mathrm{Si}$ off the grains while species such as $\mathrm{H}_{2} \mathrm{O}, \mathrm{HNCO}, \mathrm{CH}_{3} \mathrm{OH}$ can be released in milder events resulting in the icy grain mantle coming off and releasing them into the gas phase. $\mathrm{H}_{2} \mathrm{O}$ in external galaxies have been studied by Herschel (e.g. González-Alfonso et al. $2010)$. Note that $\mathrm{HCN} / \mathrm{HCO}^{+} J=(1-0)$ abundance ratios may be elevated in shocks (e.g. Kazandjian et al. 2012) even though this line ratio is not viewed as a classic shock tracer. Meijerink et al. (2013) suggest that a high CO-to-continuum ratio may be a key diagnostic for the presence of shocks.

\section{Global line ratios and spectral scans}

There are a large number of studies using global molecular line ratios to attempt to classify galaxies in terms of nuclear activity and evolutionary status (e.g. Aalto et al. 1995; Paglione et al. 2001; Aalto et al. 2002; Gao \& Solomon 2004; Graciá-Carpio et al. 2006; Krips et al. 2008; Baan et al. 2010; Papadopoulos et al. 2010). Although effects of radiative transfer and excitation are difficult to account for in these surveys, they are useful in identifying trends and searching for correlations. These relations may then be further explored with multi-transition observations as well as higher resolution studies. The new broadband receivers - mounted on interferometers such as the SMA, PdBI and ALMA, and on single dish telescopes including the IRAM 30m telescope - allow several lines to be measured simultaneously improving the accuracy of the line ratios. One such example is the EVOLUTION study on the IRAM 30m where the 3mm EMIR backend was used to observe spectral properties of 25 infrared luminous galaxies selected from the IR PAH (polycyclic aromatic hydrocarbons)-silicate diagnostic diagram of Spoon et al. (2007). Simultaneous observations of $\mathrm{HCN}, \mathrm{HCO}^{+}, \mathrm{HNC}, \mathrm{HC}_{3} \mathrm{~N}, \mathrm{C}_{2} \mathrm{H}, \mathrm{SiO}$ and $\mathrm{CO}$, ${ }^{13} \mathrm{CO}, \mathrm{C}^{18} \mathrm{O}, \mathrm{CN}$ were carried out to look for correlations Costagliola et al. (2011). The $\mathrm{HCO}^{+} / \mathrm{HCN} J=(1-0)$ ratio is correlated with the $\mathrm{PAH}$ equivalent width suggesting that there is a connection to PDRs. In general it was found that $\mathrm{HNC}$ and $\mathrm{HCO}^{+}$ $J=(1-0)$ emission appear anti-correlated (see Figure 2) - which is difficult to explain 
with standard PDR models and it is suggested that mechanical heating must be added to the models (e.g. Baan et al. 2010; Costagliola et al. 2011; Meijerink et al. 2011). All HNCbright objects are either luminous IR galaxies (LIRGs) or Seyferts. Galaxies with bright $\mathrm{PAH}$ emission show low $\mathrm{HNC} / \mathrm{HCO}^{+}$ratios. Note, however, that variations in the ratios between $\mathrm{HCN}, \mathrm{HNC}$ and $\mathrm{HCO}^{+}$are relatively small. Stronger effects are obtained for fainter species such as $\mathrm{HC}_{3} \mathrm{~N}$. The only $\mathrm{HC}_{3} \mathrm{~N}$ detections are in objects with $\mathrm{HCO}^{+} / \mathrm{HCN}$ $J=(1-0)$ intensity ratios $<1$ (see Figure 2). Galaxies with the highest $\mathrm{HC}_{3} \mathrm{~N} / \mathrm{HCN}$ ratios have warm IRAS colors $(60 / 100 \mu \mathrm{m}>0.8)$ and it is suggested that $H C_{3} N$ is a tracer of young, dust enshrouded activity.

\subsection{Spectral scans}

Combining many lines simultaneously in a spectral scan will give a more complete (and complex) picture of the chemical status of a galaxy. This includes emission from rarer species with clearer diagnostic value. Spectral scans of the nearby starburst galaxies NGC253 and M82, and the starburst/Seyfert NGC1068 have been carried out at 1, 2 and $3 \mathrm{~mm}$ wavelengths by the IRAM $30 \mathrm{~m}$ and the Nobeyama $45 \mathrm{~m}$ telescopes (e.g. Martín et al. 2006; Nakajima et al. 2011; Aladro et al. 2011, 2013). The chemistry of NGC253 shows a similarity to that of the Galactic center molecular clouds, which are thought to be dominated by low-velocity shocks. In contrast, the surveys of M82 reveal a PDR chemistry different from that of NGC253. For NGC1068 some of the carbon-chemistry appears similar to that of M82 while other aspects of the chemistry seem to be dominated by cosmic rays and XDRs. The ULIRG Arp220 has been surveyed in the $1 \mathrm{~mm}$ band by the SMA interferometer (Martín et al. 2011) and the chemical composition seems consistent with an ISM heated by a young starburst and chemically enriched by consecutive bursts of star formation. Vibrationally excited emission from $\mathrm{HC}_{3} \mathrm{~N}$ and $\mathrm{CH}_{3} \mathrm{CN}$ are indicative of a warm, intense IR field. With ALMA, a multi-band spectral scan has been carried out of the LIRG NGC4418 where the spectrum (Figure 4) is dominated by many rotationalvibrational lines of $\mathrm{HC}_{3} \mathrm{~N}$ (Aalto et al. 2007a; Costagliola \& Aalto 2010; Sakamoto et al. 2010). To date, however, the richest extragalactic scan is not towards the nucleus of a nearby galaxy but instead in the disk of a $z=0.89$ spiral galaxy intervening the continuum emission of a distant quasar (PKS1830-211). A $7 \mathrm{~mm}$ spectral survey of molecular absorption lines reveal a total of 28 different species, plus 8 isotopic variants the largest number of detected molecular species of any extragalactic object so far. The molecular fractional abundances are found to be in-between those in typical Galactic diffuse and translucent clouds, and clearly deviate from those observed in the dark cloud TMC 1 or in the Galactic center giant molecular cloud Sgr B2. The isotopic ratios of carbon, nitrogen, oxygen, and silicon deviate significantly from the solar values, which can be linked to the young age of the $z=0.89$ galaxy and a release of nucleosynthesis products dominated by massive stars (Muller et al. 2011).

\section{Molecules at high spatial resolution}

Interferometric studies provide both spatial resolution and sufficient pointing accuracy to allow us to separate regions of different dominant chemical processes. High resolution studies on IC 342 and Maffei 2 (Meier \& Turner 2005, 2012) show that the HCN, HNC, $\mathrm{HCO}^{+} J=(1-0)$ line emission and $3 \mathrm{~mm}$ continuum are spatially correlated, suggesting a close connection to star formation. In contrast, $\mathrm{HNCO}$ and $\mathrm{CH}_{3} \mathrm{OH}$ follow the molecular bar arms, especially the bar ends. This is probably caused by the spiral and bar shocks resulting in the icy grain mantles coming off. The $\mathrm{C}_{2} \mathrm{H}$ emission prefers the starburst region (but is somewhat more extended) in Maffei 2 where it is also tracing a nuclear 


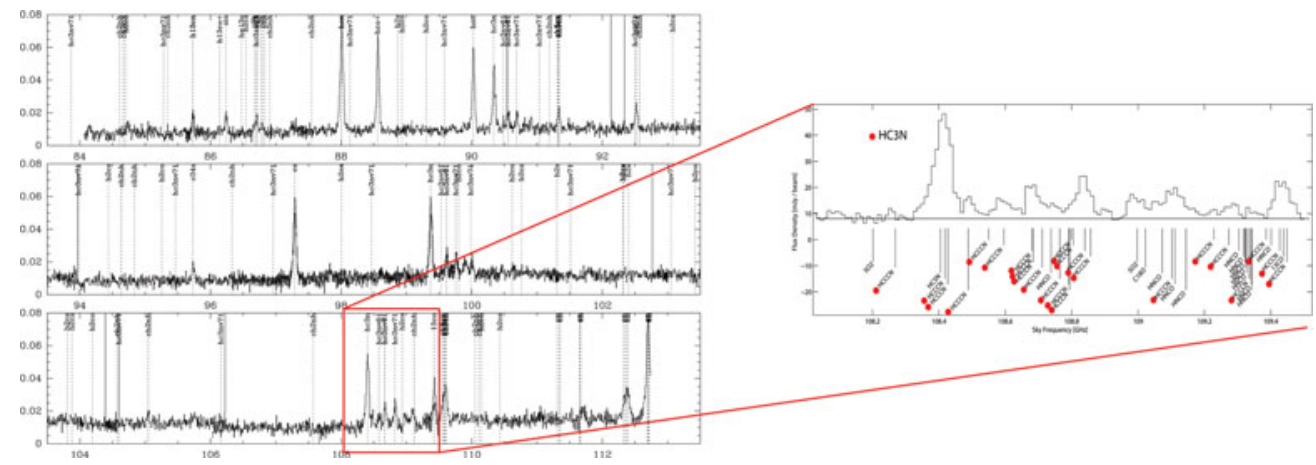

Figure 4. ALMA spectral scan of the LIRG NGC4418: Preliminary results of sections of the $3 \mathrm{~mm}$ Band 3 scan revealing the rich spectrum of NGC4418. The zoomed-in figure shows an $\mathrm{HC}_{3} \mathrm{~N}$ line forest. (Costagliola et al., in prep). [A COLOR Version is available onLine.]
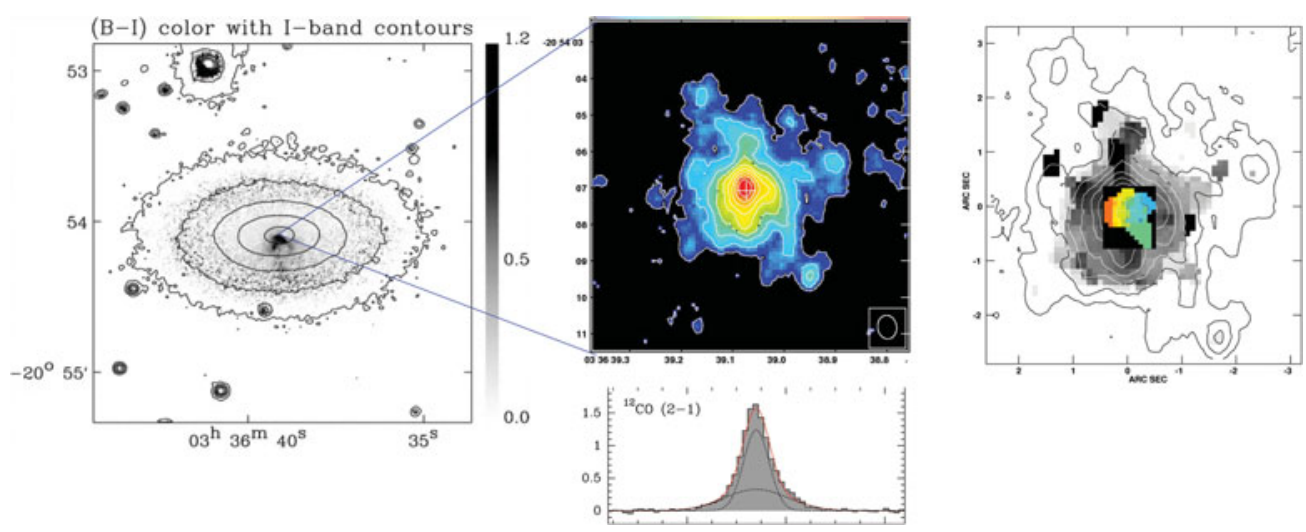

Figure 5. Left panel: Optical (B-I) image of NGC1377 from Roussel et al. (2006) showing the southern dust feature. Center panel (top): Color plot of the SMA CO $J=(2-1)$ integrated intensity towards the nucleus of NGC1377, below it we see the global CO $J=(2-1)$ profile (Aalto et al. 2012). Right panel: The CO $J=(2-1)$ integrated intensity (contours) with the dispersion map shown in grayscale. Both the integrated intensity and the dispersion map show the x-shape of a biconical outflow. The velocity field of the underlying molecular disk is shown in color. The CO $J=(2-1)$ data are consistent with a nuclear disk/outflow system. The blueshifted part of the outflow coincides with the southern dust feature. [A COLOR VERSion is AVAILABLE online.]

outflow. In IC342, $\mathrm{C}_{2} \mathrm{H}$ is instead found near the nuclear star cluster (more evolved star formation) and not the current star formation.

There have been many studies of the chemistry of the nearby Seyfert galaxy NGC1068. High resolution interferometric observations of $\mathrm{SiO}$ and CN (García-Burillo et al. 2010) reveal that $\mathrm{SiO}$ is detected in a $400 \mathrm{pc}$ circumnuclear disk (CND) around the AGN with $\mathrm{SiO}$ abundances $\left(10^{-9}\right)$ more than one order of magnitude above those measured in the starburst ring. The overall abundance of $\mathrm{CN}$ in the $\mathrm{CND}$ is also high, $10^{-7}$. Abundances measured for $\mathrm{CN}$ and $\mathrm{SiO}$ and the correlation of $\mathrm{CN} / \mathrm{CO}$ and $\mathrm{SiO} / \mathrm{CO}$ ratios with hard X-ray irradiation suggest that the CND of NGC1068 has become a giant XDR. Recent ALMA high resolution observations of $\mathrm{HCN} \mathrm{HCO}^{+} J=(4-3)$ in the CND (GarciaBurillo et al., in prep) show the ratio decreasing on small scales towards the AGN. The interpretation of this is not obvious, but it reveals that the impact of the AGN combined with effects of dynamics and physical conditions must be studied at high spatial resolution to disentangle the contributing effects. 


\subsection{Molecular excitation in galaxy nuclei}

To correctly interpret molecular line ratios it is important to separate effects of excitation from those of chemistry. In some cases this can only be done at high spatial resolution. In the ULIRG Arp220 the $\mathrm{HCN} / \mathrm{HCO}^{+} J=(1-0)$ ratio is $>1$ at 1". 6 resolution (Imanishi et al. 2007) - this is suggested to be caused either by XDR chemistry and/or by IR pumping of HCN. However, a study at even higher resolution (0!.4) and at higher frequency $(J=(3-2)$ transition $)$ of $\mathrm{HCO}^{+}$in Arp220 reveal an interesting effect: a big part of the $\mathrm{HCO}^{+}$spectrum is missing towards the eastern and western nuclei due to deep absorption toward the continuum (Sakamoto et al. 2009). The spectra are reminiscent of P-Cygni profiles and are indicative of outflowing gas from the two ULIRG nuclei. The absorption affects the global profile of $\mathrm{HCO}^{+} J=(3-2)$ and it must be taken into account when drawing any conclusions on what is causing elevated $\mathrm{HCN} / \mathrm{HCO}^{+}$intensity ratios in Arp220. Furthermore, the HNC $J=(3-2)$ profile towards the western nucleus has a prominent, narrow emission feature where $\mathrm{HCO}^{+}$shows absorption. This may constitute the first ever detection of an HNC maser - even if the amplification is weak (Aalto et al. 2009). This maser emission may be pumped by the $21.5 \mu \mathrm{m}$ continuum emission. Recently a possible methanimine $\left(\mathrm{H}_{2} \mathrm{CNH}\right)$ maser (Rickert et al. 2011) has been detected towards the same source.

For galaxies with compact molecular nuclei (CONs) it is generally true that absorption and/or pumping processes on small scales ( $\mathrm{pc}$ to tens of $\mathrm{pc}$ ) may impact the global line profile of the molecular emission. Optically thick, hot dust cores such as the one in NGC4418 (Sakamoto et al. 2013; Costagliola et al. 2013) may serve both as sources of IR photons to radiatively excite molecules and strong $\mathrm{mm} / \mathrm{submm}$ continuum sources causing absorption phenomena. ALMA will be a tremendously important tool in disentangling the effects of excitation and chemistry in LIRGs and ULIRGs.

\section{Molecular outflows}

Outflows driven by AGNs and/or starbursts represent a strong and direct mechanism for feedback that may clear central regions or the whole galaxy of fuel for future star formation or black hole $(\mathrm{BH})$ growth. Many galactic winds and outflows carry large amounts of molecular gas and dust with them. There is a growing list of examples of molecular gas in outflows including: Early type galaxies: NGC1266 (Alatalo et al. 2011), NGC1377 (Aalto et al. 2012b); Interacting starburst LIRGs: NGC3256 (Sakamoto et al. 2006; Sakamoto 2012), M82 (Nakai et al. 1987; Walter et al. 2002), NGC2146 (Greve et al. 2000; Tsai et al. 2009); NGC253 (e.g. García-Burillo et al. 2000); NGC1614 (GarciaBurillo et al. 2013, in prep.); ULIRGs and AGNs: Mrk231 (e.g. Fischer et al. 2010; Feruglio et al. 2010; Aalto et al. 2012a; Cicone et al. 2012, 2014); M51 (Matsushita et al. 2007); LIRG and ULIRG surveys: (e.g. Baan 2007; Chung et al. 2011; Sturm et al. 2011); and high redshift QSOs: SWIRE survey (e.g. Polletta et al. 2011; Nesvadba et al. 2011).

\subsection{Winds of change - the outflow of NGC137r}

NGC1377 is an extremely radio-quiet, FIR-excess lenticular galaxy. Bright, complex CO $J=(2-1)$ line emission is emerging from the inner $400 \mathrm{pc}$ and the CO $J=(2-1)$ line has wings that are tracing a kinematical component that appears to be perpendicular to the component traced by the line core. The molecular emission of NGC1377 seems to consist of a disk-outflow system. The molecular outflow/wind has an estimated mass of $M_{\text {out }}\left(\mathrm{H}_{2}\right)>1 \times 10^{7} \mathrm{M}_{\odot}$ and mass outflow rate $>8 \mathrm{M}_{\odot} \mathrm{yr}^{-1}$. The age of the proposed outflow is estimated to be $1.4 \mathrm{Myr}$, the extent to be $200 \mathrm{pc}$, and the outflow speed quite 

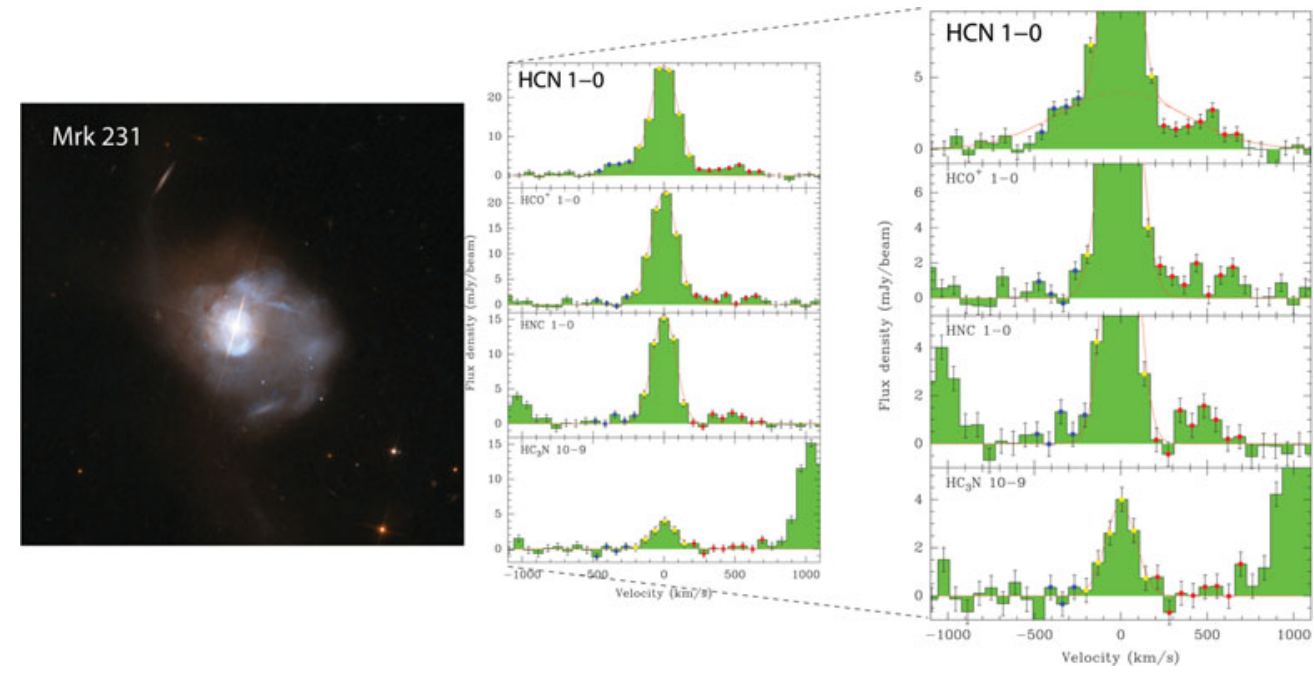

Figure 6. Left: Hubble image of Mrk231. Center and Right: Plateau de Bure interferometric spectra of $\mathrm{HCN} \mathrm{HCO}^{+} \mathrm{HNC} J=(1-0)$ and $\mathrm{HC}_{3} \mathrm{~N} J=(10-9)$. In the right panels we have zoomed in on the base of the line to show the line wings more clearly. Red solid lines show Gaussian fits to the line center line widths. In the top right panel two Gaussians are fitted (Aalto et al. 2012a). [A COLOR VERsion is AVAILABLE ONLINE.]

moderate $V_{\text {out }}=140 \mathrm{~km} \mathrm{~s}^{-1}$. The mass in the outflow is estimated to be at least $10 \%$ of the total molecular gas mass of NGC1377 (Figure 5).

The age of the outflow supports the notion that the current nuclear activity of NGC1377 is young - a few Myr. The outflow may be powered by radiation pressure from a compact, dust enshrouded nucleus, but other driving mechanisms are possible. The buried source may be an active galactic nucleus (AGN) or an extremely young (1 Myr) compact starburst. Limitations on size and mass support the AGN scenario, but more studies are necessary. In either case, the wind with its implied mass outflow rate will quench the nuclear power source within the very short time of 5-25 Myr. It is possible, however, that the gas is unable to escape the galaxy and may eventually fall back onto NGC1377 again.

\subsection{Gas properties in outflows}

Studying the physical and chemical conditions of the outflowing molecular gas will help us understand the driving mechanism, origin of the gas and its fate in the wind. Detection of bright $\mathrm{SiO}$ emission in a supershell and chimney of M82 (García-Burillo et al. 2001) show shock processed dust grain chemistry in the starburst wind. Imaging of low- $J$ CO lines in the extreme, high-velocity wind of the QSO ULIRG Mrk231 (e.g. Feruglio et al. 2010; Cicone et al. 2012) can be used to study the excitation of the lower density gas in the outflow. Interestingly, the Mrk 231 outflow has very bright $H C N J=(1-0)$ emission (Aalto et al. 2012a) (see Figure 6) and emission from $\mathrm{HNC}$ and $\mathrm{HCO}^{+} J=(1-0)$ is also detected in the outflow. Very recent PdBI imaging shows that the broad line wings are also present in the $\mathrm{HCN} J=(3-2)$ and $J=(2-1)$ spectra (Aalto et al. in prep.). The HCN $J=(1-0)$ flux actually rivals that of CO $J=(1-0)$ in the outflow - so, why is HCN so bright? There are several options: a) Large numbers of dense clumps in the outflow. b) Extremely large HCN abundances and c) mid-IR pumping of HCN. If the outflow is extended beyond the reach of an optically thick mid-IR source of at least $85 \mathrm{~K}$ then c) can be ruled out. The low CO excitation (Cicone et al. 2012) indicates that only some of the volume can be filled with dense clumps which leaves a combination of a) and 
b). High HCN abundances are expected in warm regions, e.g. in AGNs (e.g. Harada et al. 2010) or in shocks (e.g. Tafalla et al. 2010; Kazandjian et al. 2012) and a possibility is that elevated HCN luminosity is a signature of AGN-driven outflows. However, with a current sample of only one, this requires more study.

\section{References}

Aalto, S., Beswick, R., \& Jütte, E. 2010, A\& $A$ 522, A59

Aalto, S., Booth, R. S., Black, J. H., \& Johansson, L. E. B. 1995, A $\mathscr{G} A$ 300, 369

Aalto, S., Costagliola, F., van der Tak, F., \& Meijerink, R. 2011, A\& A 527, A69

Aalto, S., Garcia-Burillo, S., Muller, S., et al. 2012a, A\&A 537, A44

Aalto, S., Monje, R., \& Martín, S. 2007a, A\&SA 475, 479

Aalto, S., Muller, S., Sakamoto, K., et al. 2012b, A\& A 546, A68

Aalto, S., Polatidis, A. G., Hüttemeister, S., \& Curran, S. J. 2002, A\&A 381, 783

Aalto, S., Radford, S. J. E., Scoville, N. Z., \& Sargent, A. I. 1997, ApJ Lett. 475, L107

Aalto, S., Spaans, M., Wiedner, M. C., \& Hüttemeister, S. 2007b, A\& A 464, 193

Aalto, S., Wilner, D., Spaans, M., et al. 2009, A\&\&A 493, 481

Aladro, R., Martín, S., Martín-Pintado, J., et al. 2011, A $8 A$ 535, A84

Aladro, R., Viti, S., Bayet, E., et al. 2013 A $\& A$ 549A, 39

Alatalo, K., Blitz, L., Young, L. M., et al. 2011, ApJ 735, 88

Ao, Y., Henkel, C., Menten, K. M., et al. 2013, A\&\&A 550, A135

Baan, W. A. 2007, Nea Astr. Revs 51, 149

Baan, W. A., Loenen, A. F., \& Spaans, M. 2010, A\&A 516, A40

Bayet, E., Williams, D. A., Hartquist, T. W., \& Viti, S. 2011, MNRAS 414, 1583

Chung, A., Yun, M. S., Naraynan, G., Heyer, M., \& Erickson, N.R. 2011, ApJ Lett. 732, L15+

Cicone, C., Feruglio, C., Maiolino, R., et al. 2012, A\&A 543, A99

Cicone, C., Maiolino, R., Sturm, E., et al. 2014 A $\& A$ 562A, 21

Costagliola, F. \& Aalto, S. 2010, A\& A 515, A71

Costagliola, F., Aalto, S., Rodriguez, M. I., et al. 2011, A\&A 528, A30

Costagliola, F., Aalto, S., Sakamoto, K., et al. 2013, A $\& A$ 556, A66

Curran, S. J., Polatidis, A. G., Aalto, S., \& Booth, R.S. 2001, A\&\&A 373, 459

Evans, A. S., Becklin, E. E., Scoville, N. Z., et al. 2003, AJ 125, 2341

Feruglio, C., Maiolino, R., Piconcelli, E., et al. 2010, A\&A 518, L155+

Fischer, J., Sturm, E., González-Alfonso, E., et al. 2010, A $\xi A$ 518, L41

Gao, Y. \& Solomon, P. M. 2004, ApJS 152, 63

García-Burillo, S., Martín-Pintado, J., Fuente, A., \& Neri, R. 2000, A\&\&A 355, 499

García-Burillo, S., Martín-Pintado, J., Fuente, A., \& Neri, R. 2001, ApJ Lett. 563, L27

García-Burillo, S., Usero, A., Fuente, A., et al. 2010, A\&\&A 519, A2

González-Alfonso, E., Fischer, J., Isaak, K., et al. 2010, A 6 A 518, L43

Graciá-Carpio, J., García-Burillo, S., Planesas, P., \& Colina, L. 2006, ApJ Lett. 640, L135

Greve, A., Neininger, N., Tarchi, A., \& Sievers, A. 2000, A\&A 364, 409

Guillet, V., Jones, A. P., \& Pineau Des Forêts, G. 2009, A\&SA 497, 145

Harada, N., Herbst, E., \& Wakelam, V. 2010, ApJ 721, 1570

Helfer, T.T. \& Blitz, L. 1993, ApJ 419, 86

Hirota, A., Kuno, N., Sato, N., et al. 2010, PASJ 62, 1261

Hollenbach, D.J. \& Tielens, A.G.G.M. 1997, ARAA 35, 179

Imanishi, M. \& Nakanishi, K. 2013, $A J$ 146, 91

Imanishi, M., Nakanishi, K., Tamura, Y., Oi, N., \& Kohno, K. 2007, AJ 134, 2366

Imanishi, M., Nakanishi, K., Tamura, Y., \& Peng, C. 2009, AJ 137, 3581

Kazandjian, M. V., Meijerink, R., Pelupessy, I., Israel, F. P., \& Spaans, M. 2012, A 8 A 542, A65

Kohno, K. 2003, in Astronomical Society of the Pacific Conference Series, Vol. 289, The Proceedings of the IAU 8th Asian-Pacific Regional Meeting, Volume 1, ed. S.Ikeuchi, J.Hearnshaw, \& T.Hanawa, 349-352

König, S., Aalto, S., Muller, S., Beswick, R. J., \& Gallagher, J. S. 2013, A $\& A$ 553, A72 
Krips, M., Neri, R., García-Burillo, S., et al. 2008, ApJ 677, 262

Lepp, S. \& Dalgarno, A. 1996, A\&3A 306, L21

Lindberg, J. E., Aalto, S., Costagliola, F., et al. 2011, A 8 A 527, A150

Maloney, P. R., Hollenbach, D. J., \& Tielens, A. G. G. M. 1996, ApJ 466, 561

Mangum, J. G., Darling, J., Henkel, C., et al. 2013, ApJ 779, 33

Martin, C. L., Walsh, W. M., Xiao, K., et al. 2004, ApJS 150, 239

Martin, R. N. \& Ho, P. T. P. 1986, ApJ Lett. 308, L7

Martín, S., Krips, M., Martín-Pintado, J., et al. 2011, A\&A 527, A36

Martín, S., Mauersberger, R., Martín-Pintado, J., Henkel, C., \& García-Burillo, S. 2006, ApJS 164,450

Matsushita, S., Muller, S., \& Lim, J. 2007, A\&A 468, L49

Meier, D. S. \& Turner, J. L. 2005, ApJ 618, 259

Meier, D. S. \& Turner, J. L. 2012, ApJ 755, 104

Meier, D. S., Turner, J.L., \& Hurt, R. L. 2000, ApJ 531, 200

Meijerink, R., Kristensen, L. E., Weiß, A., et al. 2013, ApJ Lett. 762, L16

Meijerink, R. \& Spaans, M. 2005, A\&GA 436, 397

Meijerink, R., Spaans, M., Loenen, A. F., \& van der Werf, P. P. 2011, A\& A 525, A119

Mills, E. A. C., Güsten, R., Requena-Torres, M. A., \& Morris, M. R. 2013, ApJ 779, 47

Mühle, S., Seaquist, E. R., \& Henkel, C. 2007, ApJ 671, 1579

Muller, S., Beelen, A., Guélin, M., et al. 2011, A\&\&A 535, A103

Nakai, N., Hayashi, M., Handa, T., et al. 1987, PASJ 39, 685

Nakajima, T., Takano, S., Kohno, K., \& Inoue, H. 2011, ApJ Lett. 728, L38

Narayanan, D., Krumholz, M. R., Ostriker, E. C., \& Hernquist, L. 2012, MNRAS 2537

Nesvadba, N. P. H., Polletta, M., Lehnert, M. D., et al. 2011, MNRAS 415, 2359

Nomura, H. \& Millar, T. J. 2004, A\&SA 414, 409

Olsson, E., Aalto, S., Thomasson, M., \& Beswick, R. 2010, A\&JA 513, A11

Paglione, T. A. D., Wall, W. F., Young, J. S., et al. 2001, ApJS 135, 183

Papadopoulos, P. P., van der Werf, P., Isaak, K., \& Xilouris, E. M. 2010, ApJ 715, 775

Polletta, M., Nesvadba, N. P. H., Neri, R., et al. 2011, A\&A 533, A20

Rickert, M., Momjian, E., Sarma, A., \& AO Arp 220 Team. 2011, in Bulletin of the American Astronomical Society, Vol.43, American Astronomical Society Meeting Abstracts \#217, \#332.01

Rodriguez-Franco, A., Martin-Pintado, J., \& Fuente, A. 1998, A\&A 329, 1097

Sakamoto, K. 2012, ArXiv e-prints

Sakamoto, K., Aalto, S., Costagliola, F., et al. 2013, ApJ 764, 42

Sakamoto, K., Aalto, S., Evans, A. S., Wiedner, M. C., \& Wilner, D.J. 2010, ApJ Lett. 725, L228

Sakamoto, K., Aalto, S., Wilner, D. J., et al. 2009, ApJ Lett. 700, L104

Sakamoto, K., Ho, P. T. P., \& Peck, A. B. 2006, ApJ 644, 862

Schilke, P., Walmsley, C. M., Pineau Des Forets, G., et al. 1992, A $\& A$ 256, 595

Spoon, H. W. W., Marshall, J. A., Houck, J. R., et al. 2007, ApJ Lett. 654, L49

Sturm, E., González-Alfonso, E., Veilleux, S., et al. 2011, ApJ Lett. 733, L16+

Suchkov, A., Allen, R. J., \& Heckman, T. M. 1993, ApJ 413, 542

Tafalla, M., Santiago-García, J., Hacar, A., \& Bachiller, R. 2010, A\& A 522, A91

Tosaki, T., Hasegawa, T., Shioya, Y., Kuno, N., \& Matsushita, S. 2002, PASJ 54, 209

Tsai, A.-L., Matsushita, S., Nakanishi, K., et al. 2009, PASJ 61, 237

Usero, A., García-Burillo, S., Martín-Pintado, J., Fuente, A., \& Neri, R. 2007, Nea Astr. Revs 51,75

van der Tak, F. F. S., Aalto, S., \& Meijerink, R. 2008, A $6 A$ 477, L5

van der Werf, P. P., Isaak, K.G., Meijerink, R., et al. 2010, A\&A 518, L42

Viti, S. 2005, in IAU Symposium, Vol. 231, Astrochemistry: Recent Successes and Current Challenges, ed. D.C. Lis, G.A. Blake, \& E.Herbst, 67-76

Viti, S., Jimenez-Serra, I., Yates, J.A., et al. 2011, ApJ Lett. 740, L3

Wada, K. \& Tomisaka, K. 2005, ApJ 619, 93

Walter, F., Weiss, A., \& Scoville, N. 2002, ApJ Lett. 580, L21 